\title{
Using Alert and Action Expected Times of Delivery in Prevention of Prolonged Labor
}

\author{
N. F. B. Tandu-Umba*, G. Kalombo Muamba \\ Department of Obstetrics and Gynecology, University Clinics, Kinshasa, DR Congo \\ Email: "btanduumba@yahoo.fr
}

Received 12 October 2015; accepted 28 November 2015; published 1 December 2015

Copyright (C) 2015 by authors and Scientific Research Publishing Inc.

This work is licensed under the Creative Commons Attribution International License (CC BY). http://creativecommons.org/licenses/by/4.0/

c) (i) Open Access

\section{Abstract}

Background and Objective: Although globally admitted as the most valuable tool to prevent prolongation of labor, the partogram has failed to be commonly used. This is due to its alleged complexity. Based on the simplified model proposed by Debdas, the so called paperless partogram, we aimed at evaluating the ability of only using the alert and action lines to prevent prolongation of labor. Methods: This was a cross-sectional study including labor records of women delivered at King Baudouin Hospital of Kinshasa (secondary level) from 01/01 till 31/12/2013. The study was approved by the Faculty Ethical Committee. Inclusion criteria were: 1) live singleton pregnancy, 2) cephalic fetal presentation, 3) lack of uterine scar, 4) monitoring in labor ward by $4 \mathrm{~cm}$ of cervical dilation, and 5) delivery at term. For every record, the expected time of delivery (ETD $=6$ hours after $4 \mathrm{~cm}$ of cervical dilation) was considered "Alert EDT" to which 4 hours were added to obtain the "Action EDT". Irrespective of other fetal and maternal features contained in the traditional partogram Alert and Action ETD were checked a posteriori on Debdas's model to derive the appropriate outcome of labor. Results: The study included 357 participants, of which 219 primiparous and 138 multiparous. Vaginal delivery took place in $91 \%$ of cases. Full cervical dilation was achieved after 8 - 9 hours $(9.5 \pm 1.8$ hours for primiparous and $8.4 \pm 1.7$ hours for multiparous women), namely 2 - 3 hours following Alert ETD). This duration is close to the Action ETD. For 32 cesarean sections (9\%) final decision took place within the Alert ETD. Conclusion: Using only Alert and Action ETD was found convenient to derive appropriate measures for the outcome of labor. So, the paperless partogram is a simplified method to manage the active stage of labor that could prevent prolongation of labor in our setting.

\section{Keywords}

Management of Labor, Alert and Action Times of Delivery, Paperless Partogram

${ }^{*}$ Corresponding author.

How to cite this paper: Tandu-Umba, N.F.B. and Muamba, G.K. (2015) Using Alert and Action Expected Times of Delivery in Prevention of Prolonged Labor. Open Journal of Obstetrics and Gynecology, 5, 813-818. 


\section{Introduction}

The partogram is a chart of cervical dilation during labor. It was first described in the 1950s by Friedman [1] and thereafter completed by the concept of alert and action lines by Philpott and Castle [2]. The normal progression of cervical dilation includes two phases: 1 ) the first phase (latent phase) during which effacement occurs and dilation proceeds till $4 \mathrm{~cm}$ and 2) the active phase (from $4 \mathrm{~cm}$ till full dilation). The latent phase is slower and has a variable duration (6 - 8 hours) whilst the active phase proceeds at the rate of $1-1.5 \mathrm{~cm}$ per hour. According to the WHO's pattern, an alert line is plotted in the chart to correspond with the start of the active phase $(4 \mathrm{~cm})$ and extends to the point of expected full dilation $(10 \mathrm{~cm})$. The action line is plotted parallel 4 hours after the alert line. If the pattern is normal, plotting of the cervical dilation will remain on or to the left of the alert line. If it moves to the right of the alert line, approaching the action line, there is a tendency to labor prolongation. Therefore, correctives measures are to be taken. Crossing of the action line means need for cesarean section.

A long-lasting active stage of labor is known to bring a higher risk of uterine rupture, postpartum hemorrhage, maternal sepsis and death and perinatal anoxia, infection and death [3] [4]. So, the partogram has gained acceptance as one of the most valuable tools to prevent prolongation of labor, making it possible to minimize maternal-infant's adverse outcomes [3]. Although controversies have questioned its effectiveness [5]-[8] there has been global agreement that it is one of the most important advances in modern obstetric care [3] [4].

However, continuously plotting numerous variables in a pre-printed diagram and thereafter interpreting data according to cervical dilation against time elapsed require some skills and are time-consuming. This is perceived as insult to midwives' autonomy [6] [7] and flexibility [8]. This also could explain why it failed to gain adhesion of practitioners in labor wards, which seems to be its biggest challenge. In Africa, the rate of its use has been very low: $27 \%$ in Guinea [9], 25\% in Nigeria [9], 11\% in Kenya [9] and 5\% in Côte d'Ivoire [6]. Nothing has been published in DR Congo. It is common to say that it is rarely used. When used, it was rarely well interpreted and in settings where it was likely to be well interpreted it used to be fulfilled afterwards, which made it be called "post-gram" [10].

Due to its complexity consecutive simpler versions of the partogram have been designed; one of them being the Debdas's paperless partogram [11]-[13] which needs no particular training and no plotting, is free of encumbering details and suitable in low staffing facilities. It relies on the two benchmarks of the partogram: the alert and action lines. From these lines are derived the Alert expected time of Delivery (ETD) and Action ETD, the only ones to adhere to. The present study is aimed to evaluate in delivered women how only using these times might suffice to derive appropriate measures for the outcome of labor.

\section{Methods}

This was a cross-sectional study based on labor records of women delivered at King Baudouin Hospital of Kinshasa (a secondary level institution with a delivery count of 673 per year) during one year (01/01-31/12/2013). The study was scheduled as a partial fulfillment of the completion of graduation in medicine by the second author (MKG). Approval for study was obtained from the Faculty Ethical Committee and there was no need of written informed consent from each woman since files were considered for the study long after delivery. Inclusion criteria were: 1) live singleton pregnancy, 2) cephalic fetal presentation, 3) lack of uterine scar, 4) monitoring in labor ward by $4 \mathrm{~cm}$ of cervical dilation so as to expect a full dilation $(10 \mathrm{~cm})$ in 6 hours, and 5) delivery at term based on last menstrual period and ultrasound assessment at $\leq 20$ week gestation. Women with more dilated cervix at the admission and those having experienced contractions augmentation were excluded.

In this maternity unit, monitoring of labor is carried out using the partogram form designed by the Ministry of Health according to WHO's recommendations: maternal variables such as arterial blood pressure, pulse rate, uterine contractions, cervical dilation and condition of amniotic fluid, along with fetal heart rate and descent were contained in the file. Assuming that the WHO's partogram use is substandard, no attention was paid on traditional parameters of the labor management. So, in every record, the estimated time of delivery (6 hours after $4 \mathrm{~cm}$ of cervical dilation) was a posteriori considered the "Alert EDT" to which 4 hours were added to obtain the "Action EDT" at which a cesarean section might be scheduled.

Variables of study included maternal general characteristics such as age, weight, gravidity, gestational age and obstetrical ones such as time and mode of delivery (including the indication in case of cesarean section), birth weight, Apgar score at first and fifth minutes. Alert and Action ETD were checked on Debdas's model (paperless partogram) to derive proper measures according to outcome of labor. Data analysis was carried out 
using Chi-square and t-test where appropriate through the Statistical Package for Social Science (version 10.0 for Windows, SPSS). Significance was stated at p-value $<0.05$.

\section{Results}

Our study included 357 participants, of which 219 primiparous (61\%) and 138 multiparous (39\%). Their general characteristics are depicted in Table 1.

From data of this table it can be seen that the study sample was mostly primiparous and at term, making expect some women likely to experience obstructed labor.

Starting at $4 \mathrm{~cm}$ of cervical dilation, the overall mean duration to achieve full dilation was 9.1 hours (range of 8.4 - 9.5 hours), that means within 3 hours after Alert ETD (Table 2).

We observed that only 20\% of deliveries took place within the 6 hours of the Alert ETD, most of them among multiparous. The labor proceeded significantly faster in multiparous than primiparous. The overall mean duration is somewhat minimized if we take in account all cases when labor was shortened by the decision of cesarean section (acute fetal distress and severe preeclampsia) (Table 3). No uterine rupture was observed.

We also observed that, apart from 4 cases of severe preeclampsia that were diagnosed during labor, no pregnancy complication was found likely to precipitate a decision of cesarean section such as placenta prævia, abruption placentae. Cesarean section was performed $6 \pm 0.4$ hours after the beginning of active stage of labor. With regards to acute fetal distress, no detail could show an underlying cause.

Table 1. General characteristics of the study sample.

\begin{tabular}{cccc}
\hline Variables & Means & SD \\
\hline Age (years) & 26.4 & 1.6 \\
Gravidity & 3.3 & 0.5 \\
Parity & 2.2 & 0.4 \\
Weight (kg) & 55.8 & 1.7 \\
Gestational age (weeks) & 37.8 & 1.2 \\
\hline
\end{tabular}

Table 2. Mean duration of the active stage of labor.

\begin{tabular}{ccccc}
\hline \multirow{2}{*}{ Duration (hours) } & Primiparous & Multiparous & Total & \multirow{2}{*}{$\mathrm{p}$} \\
\cline { 2 - 4 } & $\mathrm{N}(\%)$ & $\mathrm{N}(\%)$ & $\mathrm{N}(\%)$ & \\
\hline $6-7$ & $8(2.2)$ & $63(17.7)$ & $71(19.9)$ & $<0.0001^{*}$ \\
$8-9$ & $194(54.3)$ & $73(20.5)$ & $267(74.8)$ & $<0.0001^{*}$ \\
$10-11$ & $17(4.7)$ & $2(0.6)$ & $19(5.3)$ & $0.06^{*}$ \\
Overall duration & $9.5 \pm 1.8$ & $8.4 \pm 1.7$ & $9.1 \pm 1.2$ & $<0.0001^{* *}$ \\
${ }^{*} \chi^{2} ;{ }^{* *}$ t-test. & & & & \\
\end{tabular}

Table 3. Characteristics of deliveries.

\begin{tabular}{|c|c|c|}
\hline Mode of delivery & $\mathbf{N}$ & $\%$ \\
\hline Vaginal & 325 & 91 \\
\hline Cesarean section & 32 & 9 \\
\hline Obstructed labor & 11 & 3 \\
\hline Acute fetal distress & 17 & 5 \\
\hline Severe preeclampsia & 4 & 1 \\
\hline Birth weight (g) & $2904 \pm 201.2$ & - \\
\hline Apgar score $1^{\text {st }}$ minute & $8.8 \pm 1.0$ & - \\
\hline Apgar score $5^{\text {th }}$ minute & $9.7 \pm 1.0$ & - \\
\hline
\end{tabular}




\section{Discussion}

The paperless partogram uses two times to advise practitioners to terminate labor or transfer the parturient to more equipped institution for cesarean section. According to Debdas [11], both times are to be ostensibly noted (in big/bold letters) on the so-called paperless partogram such as to be seen by every caregiver. If delivery does not occur by the Alert EDT in a poor staffed/equipped setting, practitioners will be expected to refer [11]-[13]. Finally, reaching the Action EDT means a high risk of prolonged labor and needs a proper medical or surgical (cesarean section) option. All these measures do not exclude specific decisions generated by the assessment of fetal heart rate, condition of amniotic fluid, uterine contractions, fetal head position and descent etc that could precipitate a cesarean section [11]-[15].

Very few authors assessed the effectiveness of paperless partogram in the management of labor. So far, only two experimental studies are to be mentioned. Fatou et al. [16] in Egypt assessed maternal and neonatal outcomes such as mode of delivery, birth outcome, birth weight, Apgar score, and any other complications among 100 women. Mean Apgar score after 5 minutes was 9.4. There was no complication of labor. Mean duration for delivery after Alert ETD was $3.5 \pm 2.1$ hours in primigravida and $3.3 \pm 2.1$ hours in multipara. They concluded that the paperless partogram was effective on the management of labor. The main feature was: one does not need to plot many others parameters in the chart to properly advise caregivers.

Agarwal et al. [12] in India recruited 91 women, of which 13\% of induced labor. In an experimental model, they monitored parturients on the basis of Alert estimated time of delivery (ETD) and Action ETD. At the time of the Action ETD, if delivery had not yet occurred, a diagnosis of abnormal labor was made and arrangements were made for emergency obstetric care. The mean duration for delivery after Alert ETD was 4.3 hours that was similar to the World Health Organization's (WHO) recommendation for partograms, with a four-hour action line. They thus concluded that the paperless partogram was convenient and effective in the management of labor.

Our study is the first one dealing with assessment of the efficacy of the paperless partogram without performing prospective/experimental evaluations. Our data were collected from already delivered women, without special attention paid on individuals with a specific characteristic. Assuming that the WHO's partogram is poorly used in our setting, we assessed conditions emerging if only alert and action expected times of delivery were used. We thus derived how this might suffice to get outcomes encountered in each case.

With 61\% of primiparous and having a neonate with a mean weight of $2904 \pm 201.2 \mathrm{~g}$, our sample was fit for the study purpose since prolongation of labor due to obstructed labor is more likely to occur in this group of women. In our series, the mean duration of the overall active stage of labor (including both Alert and Action ETD) was $9.1 \pm 1.2$ hours, meaning that, for the majority of women, 3 extra hours are actually needed over the 6 hours conventionally expected for the completion of cervical dilation. This clearly corresponds with the fact that $80 \%$ of deliveries took place between Alert and Action ETD. This finding is in accordance with the recommendation of WHO plotting the action parallel 4 hours after the alert line [3] [4]. Such a timing of intervention was also put forth by Lavender et al. [6] and Agarwal et al. [12] who reported an average of $4.3 \mathrm{~h}$ (4.7 h for primiparous and $3.7 \mathrm{~h}$ for multiparous). In the present study we registered 32 cesarean sections (9\%). All were performed not later than $6 \mathrm{~h} 30$ after start of the active stage, the essential of their indications (11 cases of obstructed labor and 17 of acute fetal distress) likely to be linked to prolongation of labor. So final decision took place within Alert ETD. This is supportive of the recommendation that cesarean section should be decided not later than Alert ETD in order to achieve a good status of the neonate as attested by the Apgar scores of the series.

Although the WHO's pattern of partogram presently in use is assumed to be the best of multiple consecutive versions recommended since its first proposal in 1994, there is evidence that it is not easy to use. It remains encumbering and has been suffering from low rates of completion and proper interpretation [5]-[8] [11]-[13]. For long, the only barrier perceived by WHO to its use has been lack of adequate trained/educated staff. In his 2009's review dealing with studies that addressed the effect of partogram use on outcomes of spontaneous labour at term, Soni [3] stated that there was no evidence to change the current pattern and use of partogram. In 2011, the "Regional Meeting on the acceleration of partograph use in East, Central and South Africa" [14] considered that promoting the presence of skilled and motivated personnel during most of deliveries would solve the problem. It thus suggested a strategy based on ongoing education of physicians, nurses and midwives. In 2013 Bah et al. [9] concluded likewise. Asibong et al. [15] recently recommended promoting usage of this tool by staging periodic training, making partograms available in labor wards and provisioning staff numbers etc. So, 
the question of its further simplification seems to be closed. Meanwhile, training or education initiatives to promote its use have failed to achieve the goal of establishing it as an indispensable mean for labor management.

Proposal of restricting the management of labor to only two times might raise fear for leaving parturient unattended till Alert or Action ETD. This is supportive of including some extra features in the paperless partogram, e.g. fetal heart rate, vaginal examination and condition of amniotic fluid when rupture of membranes occurs. The fact that $53 \%$ of cesarean sections in our series were performed for fetal distress gives consistence to such a recommendation. It is, however, to be wondered if this extra features might lower enthusiasm that was demonstrated among practitioners who were invited to implement the paperless partogram [16]. So, the Debdas's model should be adopted in labor wards of settings where caregivers are low-skilled and/or staffed.

The strength of this study lies in the same condition that bases its weakness: the retrospective nature of data collection that avoids an experimental (interventional) design. Analyzing situations actually experienced by women in usual conditions of labor makes the study free of bias generated by awareness of persons participating in prospective evaluations along with the necessity to intervene accordingly when substandard events are encountered.

The main weaknesses of our study could lie in the fact that the paperless partogram was assessed without actually implementing it. This is basis for lack of findings on possible corrective measures taken during labor such as artificial rupture of membranes and time elapsed between the latter and delivery. These details could probably modify the overall profile of cases included in the study. Such a limitation might make practitioners cautious in implementation of the technique. Furthermore, particular patterns are to be drawn for women having particular pre-pregnancy risk factors and those delivering under conditions that constituted our exclusion criteria: multiple pregnancy, breech presentation, uterine scar, preterm delivery and induction of labor.

\section{Conclusion}

Using the Alert and Action ETD was found convenient to derive appropriate measures for the outcome of labor. So, the paperless partogram is a simplified method to manage the active stage of labor that needs advocacy among caregivers, mostly in low-skilled and/or staffed settings.

\section{Conflict of Interest}

None.

\section{Contribution of the Authors}

TUB generated the idea, designed the study and drafted the manuscript. KMG collected the data, participated in the analysis of results and participated in drafting the manuscript. The work was presented by KMG in April 2015 as a partial fulfillment of the completion of the graduation in medicine.

\section{References}

[1] Friedman, E.A. (1955) Primigravid Labour. A Graphicostatistical Analysis. Obstetrics and Gynecology, 6, 567-589. http://dx.doi.org/10.1097/00006250-195512000-00001

[2] Philpott, R.H. and Castle, W.M. (1972) Cervicographs in the Management of Labour in Primigravidae. International Journal of Obstetrics and Gynecology, 79, 592-598. http://dx.doi.org/10.1111/j.1471-0528.1972.tb14207.x

[3] World Health Organization (1994) World Health Organization Partograph in Management of Labour. Lancet, 343, 1399-1404.

[4] Tayade, S. and Jadhao, P. (2012) The Impact of Use of Modified WHO Partograph on Maternal and Prenatal Outcome. International Journal of Biomedical Advanced Research, 3, 256-262. http://dx.doi.org/10.7439/ijbar.v3i4.398

[5] Walraven, G.E. (1994) WHO Partograph. Lancet, 344, 617. http://dx.doi.org/10.1016/S0140-6736(94)92004-4

[6] Lavender, T., Hart, A. and Smyth, R.M.D. (2008) Effect of Partogram Use on Outcomes for Women in Spontaneous Labour at Term. Cochrane Database of Systematic Reviews, 8, 16. http://dx.doi.org/10.1002/14651858.cd005461.pub2

[7] Soni, B.L. (2009) Effect of Partogram Use on Outcomes for Women in Spontaneous Labour at Term: RHL Commentary. The WHO Reproductive Health Library, World Health Organization, Geneva.

[8] Lavender, T. and Malcolmson, L. (1999) Is the Partogram a Help or a Hindrance? An Exploratory Study of Midwives' Views. Practice for Midwife, 2, 23-27. 
[9] Bah, E., Camara, H., Dao, B., Aribot, J., Hyjaz, Y. and Pleah, T. (2013) Partogram: Quality of Use and Caregivers' Opinion in Guinea. 1st African Congress of FIGO, Addis Ababa, 3-7 October 2013.

[10] Kanagema, J.D. and Levin, K. (2008) Use of the Partograph: What Do We Know? What Do We Need to Find out? USAID Presentation.

[11] Debbas, A.K. (2008) Paperless Partogram. 41th Annual Scientific Sessions of Sri Lanka College of Obstetricians and Gynaecologists, 1, 124.

[12] Agarwal, K., Agarwal, L., Agarwal, V.K., Agarwal, A. and Sharma, M. (2013) Evaluation of Paperless Partogram as a Bedside Tool in the Management of Labor. Journal of Family Medical and Primary Care, 2, 47-49. http://dx.doi.org/10.4103/2249-4863.109944

[13] Adler, E. and Wirth, M. (2012) The Paperless Partogram: A Simplified Tool to Prevent Prolonged Labor.

[14] (2011) The Regional Meeting on the Acceleration of Partograph Use in East, Central and South Africa (ECSA), Nairobi, 11-12 May 2011.

[15] Asibong, U., Okokon, I.B., Agan, T.U., Oku, A., Opiah, M., Essien, J. and Monjok, E. (2014) The Use of the Partograph in Labor Monitoring: A Cross-Sectional Study among Obstetric Caregivers in General Hospital, Calabar, Cross River State. Nigeria International Journal of Women's Health, 6, 873-880.

[16] Fatouh, E. and Ramadan, S. (2015) Effect of Using Paperless Partogram on the Management and Outcome of Labour and the Nurses' Opinion. Journal of Education and Practice, 6, 47-49. 